\title{
On "Schwinger Mechanism for Gluon Pair Production in the Presence of Arbitrary Time Dependent Chromo-Electric Field"
}

\author{
S.P. Gavrilov*and D.M. Gitman ${ }^{\dagger}$
}

October 2, 2018

\begin{abstract}
Recently the paper "Schwinger Mechanism for Gluon Pair Production in the Presence of Arbitrary Time Dependent Chromo-Electric Field" by G. C. Nayak was published [Eur. Phys. J. C 59, 715 (2009); arXiv:0708.2430. Its aim is to obtain an exact expression for the probability of non-perturbative gluon pair production per unit time per unit volume and per unit transverse momentum in an arbitrary time-dependent chromo-electric background field. We believe that the obtained expression is open to question. We demonstrate its inconsistency on some well-known examples. We think that this is a consequence of using the so-called "shift theorem" arXiv:hep-th/0609192 in deriving the expression for the probability. We make some critical comments on the theorem and its applicability to the problem in question.

PACS numbers:11.15.-q,11.15.Tk
\end{abstract}

I. In a recent paper [1], an expression for $\frac{d W}{d^{4} x d^{2} p_{T}}$, the probability of non-perturbative gluon pair production per unit time per unit volume and per unit transverse momentum in an arbitrary timedependent chromo-electric background field $E^{a}(t)$ with arbitrary color index $a=\overline{1,8}$ in $S U(3)$, was represented. We believe that the obtained expression is open to question.

The main assertion of the paper [1] reads: "We find that the exact result for non-perturbative gluon pair production is independent of all the time derivatives $d^{n} E^{a} / d t^{n}$, where $n \in \mathbb{N}$, and has the same functional dependence on two Casimir invariants $C_{1}=E^{a}(t) E^{a}(t)$ and $C_{2}=$ $\left[d_{a b c} E^{a}(t) E^{b}(t) E^{c}(t)\right]^{2}$ as the constant chromo-electric field $E^{a}$ result with the replacement: $E^{a} \rightarrow E^{a}(t)$." The result is presented by Eqs. (1-2) from [1] as follows:

$$
\frac{d W_{g(\bar{g})}}{d t d^{3} x d^{2} p_{T}}=\frac{1}{4 \pi^{3}} \sum_{j=1}^{3}\left|g \Lambda_{j}(t)\right| \ln \left[1+\exp \left(-\frac{\pi p_{T}^{2}}{\left|g \Lambda_{j}(t)\right|}\right)\right],
$$

where $\Lambda_{j}(t)$ is expressed via Casimir invariants $C_{1}$ and $C_{2}$. Here $W_{g(\bar{g})}=2 \operatorname{Im} S^{(1)}$ is imaginary part of the one loop (or gluon pair) effective action $S^{(1)}$. The field $E^{a}(t)$ acts along the $z$-axis, the potential $A_{3}^{a}=0$, such that $A_{\mu}^{a}=-\delta_{\mu 0} E^{a}(t) z$.

First, one can see that eq. (11) is in contradiction with well-known and well-justified results obtained previously. To this end it is enough to consider an Abelian-like background, in which the chromo-electric field has only one nonzero component, $E^{a}(t)=\delta_{a 0} E(t)$. Then $C_{1}=[E(t)]^{2}$ and $C_{2}=0$, which implies $\Lambda_{1}(t)=|E(t)|$ and $\Lambda_{2,3}(t)=|E(t)| / 2$. If so, then it follows from eq. (11) that $W_{g(\bar{g})}$ depends in the general case (in an arbitrary field $\left.E(t)\right)$ on the time $t$. In turn, this implies (and this is an exact result according to the author) that the probability (1) is a local

\footnotetext{
*Department of General and Experimental Physics, Herzen State Pedagogical University of Russia, Moyka emb. 48, 191186 St. Petersburg, Russia; e-mail: gavrilovsergeyp@yahoo.com

${ }^{\dagger}$ Institute of Physics, University of São Paulo, CP 66318, CEP 05315-970 São Paulo, SP, Brazil; e-mail: gitman@dfn.if.usp.br
} 
quantity of which the value at the time-moment $t$ is determined by the electric $E(t)$ at the same time moment. In the case under consideration (Abelian-like background) calculations in QCD are quite analogous to that in QED. That is why one can consider similar calculations in QED, where they can be performed exactly, e.g., for the electric field of the form $E(t)=E_{0} \cosh ^{-2}(t / \alpha)$, where $E_{0}$ and $\alpha$ are some positive constants. Such a field switches on and off adiabatically at $x^{0} \rightarrow \pm \infty$. First, differential mean numbers $\aleph_{m}$ of particles created by such a field were found in [2]; see also [3, 4]). The explicit form of $\aleph_{m}$ is not important for the further consideration. What we only need to stress that these quantities depend essentially on $\alpha$, they have an essentially different form in a constant electric field, and they do not depend on time $t$ at all. The latter fact is a general property of $\aleph_{m}$ for any external field, since they are defined via an inner product of solutions of a corresponding wave equation conserved in time. Using the general formulation (see [3, 4]), one can see that, in an arbitrary time-dependent but uniform external field, the probability $P^{v}$ of there occuring no actual pair creation in the history of the field in the volume $V$ can be represented as

$$
P^{v}=\left|c_{v}\right|^{2}=\exp \left\{-2 \operatorname{Im} S^{(1)}\right\}=\exp \left\{\kappa \sum_{m} \ln \left(1-\kappa \aleph_{m}\right)\right\},
$$

where $\kappa=-1$ for bosons and $\kappa=+1$ for fermions, and the summation over $m$ implies an integration over momenta and a summation over some discrete quantum numbers. Here $c_{v}=\langle 0$, out $| 0$, in $\rangle=$ $\exp \left(i S^{(1)}\right)$ is the transition amplitude from the initial vacuum $\mid 0$, in $\rangle$ to the final vacuum $\mid 0$, out $\rangle$. Passing from the summation over momenta to the integration, $\sum_{\mathbf{p}} \ldots \rightarrow(2 \pi)^{-3} V \int d \mathbf{p} \ldots$, there appears a natural volume dependence for the uniform external field in (2), $\operatorname{Im} S^{(1)} \sim V$. However, a time dependence cannot appear in such a way in (2) (explicit expressions can be found in [5, 6]). That is why the quantity $\frac{d W_{g(\bar{g})}}{d t d^{3} x d^{2} p_{T}}$ makes no sense. The quantities $\aleph_{m}$ can also be found exactly in the case when the electric field has an exponential fall-off, $E\left(x^{0}\right)=E \exp \left(-x^{0} / \alpha\right)$ [7], and in the case of a periodic alternating electric field 8 . Then one can come to similar conclusions.

Likely, an illusion that the quantity $\operatorname{Im} S^{(1)}$ depends on time is related to the well-known Schwinger formula $\operatorname{Im} S^{(1)} \sim V T$ (see [9]), where, however, $T$ is a total effective time of a (quasi)constant electric field action. Such a result holds only for a quasiconstant electric field that switches on at $-T / 2$ and switches off at $T / 2$, being quasiconstant for the total time $T$ of the field action. If the time $T$ is sufficiently large $(\sqrt{|q E|} T \gg 1$ for massless particles $)$ then $\aleph_{m}$ is constant for sufficiently large region of $p_{3}$ and the integral over $p_{3}$ turns out to be proportional to $T, \int d p_{3} \aleph_{m} \sim|q E| T \aleph_{m}$; see [5]. We stress that in this case the quantity $\operatorname{Im} S^{(1)}$ depends on the total time $T$ of the field action, this quantity is a global one, and $d \operatorname{Im} S^{(1)} / d T$ is constant. One ought also to mention that interpretation of the quantity $\frac{d W}{d^{4} x d^{2} p_{T}}$ (given in [1]) as the probability is inaccurate in the general case (the quantity $2 \operatorname{Im} S^{(1)}$ can be interpreted as the total probability of pair creation only if the WKB approximation is applicable; see, e.g., Sect. 3.2 [10]).

Thus, one can see that something is wrong with the alleged exact result (1). Checking the derivation of eq. (11), given in 11, we have discovered that the transformation of the effective action $S^{(1)}$ from Eq.(36) to Eq.(37) is erroneous. This makes the result (1) erroneous. Doing this transformation, authors of [1] refer to the article [1], where such a transformation (named a "shift theorem") was justified (see a comment on the shift theorem below).

II. The transformation from Eq.(36) to Eq.(37) given in [1], repeats, in fact, the main assertion of the shift theorem, which in its original form in [11] is not related to peculiarities of the $\mathrm{SU}(3)$ group. One can see that this transformation acts on the matrix element

$$
\int_{-\infty}^{+\infty} d z\left\langle z\left|e^{-i s\left[\left(i \partial_{t}-g \Lambda(t) z\right)^{2}-\hat{p}_{z}^{2}-2 i g \lambda_{l} \Lambda(t)\right]}\right| z\right\rangle
$$

only, at fixed values of other variables, including color indices. Here $\lambda_{l}$ are eigenvalues of the Lorentz matrix, $\left(\lambda_{1}, \lambda_{2}, \lambda_{3}, \lambda_{4}\right)=(1,0,-1,0), \Lambda^{a b}(t)=i f^{a b c} E^{c}(t)$. Denoting, $g \Lambda(t)=a(t)$, $-2 i g \lambda_{l} \Lambda(t)=c(t)$, and $-\hat{p}_{z}^{2}=b\left(\partial_{z}\right)$, one can see that the transformation from Eq.(36) to Eq.(37) of [1] is equivalent to the assertion of the shift theorem. 
Let us denote coordinate operators by $\hat{z}$ and $\hat{t}$, and their eigenvalues by $z$ and $t$, respectively, $\hat{z}|z\rangle=z|z\rangle, \hat{t}|t\rangle=t|t\rangle$, such that $\left\langle z \mid z^{\prime}\right\rangle=\delta\left(z-z^{\prime}\right),\left\langle t \mid t^{\prime}\right\rangle=\delta\left(t-t^{\prime}\right)$. Then the main assertion (4) of the shift theorem from [11] is equivalent to the validity of the following relation:

$$
\begin{aligned}
& \int_{-\infty}^{+\infty} d z f_{1}(\hat{t})\left\langle z\left|e^{-u\left[\left(\hat{P}_{t}-a(\hat{t}) \hat{z}\right)^{2}-\hat{P}_{z}^{2}+c(\hat{t})\right]}\right| z\right\rangle f_{2}(\hat{t}) \\
& =\int_{-\infty}^{+\infty} d z f_{1}(\hat{t})\left\langle z\left|e^{i a^{-1}(\hat{t}) \hat{P}_{t} \hat{P}_{z}} e^{-u\left[a^{2}(\hat{t}) \hat{z}^{2}-\hat{P}_{z}^{2}+c(\hat{t})\right]} e^{-i a^{-1}(\hat{t}) \hat{P}_{t} \hat{P}_{z}}\right| z\right\rangle f_{2}(\hat{t}),
\end{aligned}
$$

where the operators $\hat{P}_{z}$ and $\hat{P}_{t}$ satisfy the following commutation relations $\left[\hat{P}_{z}, \hat{z}\right]=\left[\hat{P}_{t}, \hat{t}\right]=i$, and the gauge conditions

$$
\left\langle z\left|\hat{P}_{z}\right| z^{\prime}\right\rangle=-i \partial_{z}\left\langle z \mid z^{\prime}\right\rangle=i \partial_{z^{\prime}}\left\langle z \mid z^{\prime}\right\rangle,\left\langle t\left|\hat{P}_{t}\right| t^{\prime}\right\rangle=-i \partial_{t}\left\langle t \mid t^{\prime}\right\rangle=i \partial_{t^{\prime}}\left\langle t \mid t^{\prime}\right\rangle
$$

To literally reproduce the matrix element (3), one has to set $u=i s$. The proof of the shift theorem in [11] is based on a variable change which allows one to pass from eq. (23) to eq. (25) and then to (26). Moreover, the transition from eq. (23) to eq. (25) is based on the supposition that a change of the integration path over the new variable $z^{\prime}$ is possible. In our notation, such a variable change is $z=z^{\prime}+a^{-1}(\hat{t}) \hat{P}_{t}$, and it implies introducing a new coordinate operator $\hat{z}^{\prime}=\hat{z}-a^{-1}(\hat{t}) \hat{P}_{t}$. However, if $a(t)$ really depends on time, then $\left[a(\hat{t}), a^{-1}(\hat{t}) \hat{P}_{t}\right] \neq 0$ and, therefore, $\left[a(\hat{t}), \hat{z}^{\prime}\right] \neq 0$. In addition, $z^{\prime}=z-a^{-1}(\hat{t}) \hat{P}_{t}$ is not a $c$-number, since $\left[a(\hat{t}), z^{\prime}\right] \neq 0$.

It is implicitly supposed in [1] that for any $u$ (the parameter $u$ takes all the values on the real or imaginary semiaxis) the kernel in the integral on the left hand side (4) has necessary analytical properties for a change of the integration path. At the same time, it is supposed that $\left[a(\hat{t}), z^{\prime}\right]=0$ and $z^{\prime}$ is transformed as a usual coordinate $z$ on this new path. Rigorous justification for this is not given; only an analogy with Gaussian integral (7) from [11] is mentioned. Let us note that in the general case, $a(\hat{t})$ and $c(\hat{t})$ arbitrary, the kernel on the left hand side (4) as a function of $z$ is unknown due to the presence of the operator $\hat{P}_{z}^{2}$ in the exponential. Thus, such an analogy does not work. We believe that such a delusion is a result of the ambiguous notation, in which the coordinate operator and its eigenvalue are denoted by the same letters. Therefore, the transformation, used in 11 does not exist, so that the shift theorem does not hold. Consequently, one can believe that a number of results obtained in [12, 13] and based on this theorem are open to question. In the particular case that is discussed in [1] one has $a(t)=g \Lambda(t), c(t)=-2 i g \lambda_{l} \Lambda(t)$. If the field $\Lambda(t)$ really depends on time, which is the case, then $\left[\Lambda(t), i \Lambda^{-1}(t) \partial_{t}\right] \neq 0$, and the transformation from Eq.(36) to Eq.(37), given in [1], does not exist.

Acknowledgement D.M.G. acknowledges the permanent support of FAPESP and CNPq.

\section{References}

[1] G. C. Nayak, arXiv:0708.2430 Eur. Phys. J. C 59, 715 (2009).

[2] N.B. Narozhny and A.I. Nikishov, Sov. J. Nucl. Phys. (USA) 11 (1970) 596.

[3] E.S. Fradkin and D.M. Gitman, Fortschr. Phys. 29, 381 (1981); E.S. Fradkin, D.M. Gitman and S.M. Shvartsman, Quantum Electrodynamics with Unstable Vacuum (Springer-Verlag, Berlin 1991).

[4] A.A. Grib, S.G. Mamaev, and V.M. Mostepanenko, Vacuum Quantum Effects in Strong Fields (Atomizdat, Moscow 1988; Friedmann Laboratory Publishing, St. Petersburg 1994).

[5] S.P. Gavrilov and D.M. Gitman, Phys. Rev. D 53, 7162 (1996). 
[6] G.Dunne and T. Hall, Phys. Rev. D 58, 105022 (1998).

[7] B.L. Spokoiny, Sov. J. Nucl. Phys. 36 (1982) 277; Phys. Lett. A 88 (1982) 328.

[8] N.B. Narozhny and A.I. Nikishov, Sov. Phys. JETP 38, 427 (1974); V.M. Mostepanenko and V.M. Frolov, Sov. J. Nucl. Phys. (USA) 19, 451 (1974).

[9] J. Schwinger, Phys. Rev. 82, 664 (1951).

[10] S.P. Gavrilov, D.M. Gitman, and J.L. Tomazelli, Nucl. Phys. B 795, 645 (2008).

[11] F. Cooper, G. C. Nayak, arXiv:hep-th/0609192

[12] F. Cooper, G. C. Nayak, arXiv:hep-th/0611125, arXiv:hep-th/0612292.

[13] G. C. Nayak, arXiv:0705.0005 JHEP 0903, 051 (2009); arXiv:0705.2770; arXiv:0807.4319. 